\title{
Chakras' Energy Deficiencies in Patient with Congestive Heart Failure
}

\author{
Huang Wei Ling* \\ Infectious Diseases, Medical Acupuncture and Pain Management Clinic, Brazil
}

*Corresponding author: Huang Wei Ling, Infectious Diseases, Medical Acupuncture and Pain Management Clinic, São Paulo, Brazil.

\section{Received Date: October 07, 2021}

Published Date: November 12, 2021

\begin{abstract}
Statement of the problem: Congestive Heart failure (CHF) is a syndrome characterized by the inability of the Heart to bomb adequate amount of Blood to reach the necessity of each organ and system. In traditional Chinese medicine (TCM), CHF can be due to deficiency of Yang, Qi, Blood stasis, Yin deficiency, retention of water and Phlegm.

Purpose: to demonstrate that patients with CHF have chakras' energy centers deficient in energy that is leading to inability of the Heart normal function and the replenishment of these deficiencies can lead to the improvement of the function of the Heart.

Methods: one case report: 82 years-old women with Alzheimer's disease and congestive heart failure since 2012, in use of medications to control the function of the Heart, antihypertensive and antidepressant medications The family took her to acupuncture sessions to try another way of treatment of her depression and Alzheimer's disease and to treat a pain in the upper limbs. I did the radiesthesia procedure to evaluate the degree of her energy deficiency and discovered that all her chakras' energies centers (Heart, Spleen, Lung, Kidney, Liver) were in the minimum level, with exception of the seventh that was normal. In the first appointment, the patient was presenting shortness of breath when placed lying down for physical examination and had a purplish color at the fingertips and lips. Treatment started with Chinese dietary counselling, auricular acupuncture with apex ear bloodletting and replenishment of the chakras' energy centers with homeopathies according to the theory Constitutional Homeopathy of the Five Elements Based on Traditional Chinese Medicine and crystal-based medications.
\end{abstract}

Results: after the first acupuncture session and the beginning of the use of medications (homeopathies and crystals), the patient improved very quickly of her dyspnea and her purpleness on the fingertips and lips disappeared completely after beginning of the treatment.

Conclusion: patients with congestive Heart failure have energy deficiencies in the chakras' energies centers and the treatment correcting the energies disturbances (using Chinese dietary counselling, auricular acupuncture, and apex ear bloodletting, replenishing the chakras' energies centers using homeopathies medications) are very important tools in the treatment of the cause of CHF and not just treating the patients symptoms.

Keywords: Congestive heart failure; Energy; Chakras; Traditional Chinese medicine; Diet; Acupuncture; Homeopathy.

\section{Introduction}

Congestive Heart failure (CHF) is a clinical syndrome caused by structural and functional defects in myocardium resulting in impairment of ventricular filling or the ejection of Blood. The most common cause for $\mathrm{CHF}$ is reduced left ventricular myocardial function; however, dysfunction of the pericardium, myocardium, endocardium, heart valves or great vessels alone or in combination is also associated with CHF [1]. The clinical presentation of CHF comprises symptoms of shortness of breath, dyspnea; orthopnea on lying own; paroxysmal nocturnal dyspnea; fatigue, weakness, lethargy; edema, abdominal distention and right hypochondriac pain. CHF has primarily been recognized as a disease of the elderly population ( $>60$ years) and is reported to affect about $2 \%-3 \%$ of people in the United States [2]. To write this article, I will use the commandments by Hippocrates ( 460 bce - 375 bce), father of medicine, that said that we need to consider other ancient medical traditions prior to the knowledge we have nowadays, so I will use concepts of traditional Chinese medicine to explain the energy alterations that is leading to the formation of congestive heart failure [3]. 
According to the study done by Li et al (2016) entitled Chinese medicine syndromes in congestive heart failure: A literature study and retrospective analysis of clinical cases, they study 6.799 cases of patients with CHF and they demonstrated that Qi deficiency was the most affected energy deficiency in patients with CHF specially involving Heart-Qi-deficiency and secondly when the patient has Heart $Q i$ and Yin deficiency. The other energy alterations found in some patients with CHF are Blood stasis and deficiency and obstruction of Yin and Qi syndrome (in females) and Phlegm blocking Heart vessels and Blood stasis associating with Qi deficiency (most in male patients) [4].

\section{Purpose}

The purpose of this study is to demonstrate that patient that has congestive heart failure diagnosis has chakras' energy centers deficient in energy and the treatment of these energy deficiencies can improve the clinical condition of the patient with congestive heart failure, improving their quality of life, less dyspnea, less purpleness and the general improvement of their physical and emotional state condition.

\section{Method}

I did an extensive search for articles in Western medicine and in traditional Chinese medicine concepts regarding congestive Heart failure indexed in PubMed and used one case report to demonstrate how is the process of energy imbalances investigation of patient with congestive heart failure and how could be the treatment and the results, in the energy point of view.

\section{Case Report}

It is a 82 years-old-women with Alzheimer's disease, congestive Heart failure since 2012. This patient was in use of medications to control the function of the Heart, antihypertensive and antidepressant medications. This patient was complaining of pain in the right arm and the family decided to take her to do some acupuncture sessions and also try another way of treatment for her depression and Alzheimer's disease and not only to treat the pain that the patient was presenting in the upper limbs. I did the radiesthesia procedure to evaluate the degree of her energy deficiency and discovered that all her chakras' energies centers (from one to seven) were in the lowest level of energy (rated in one) with exception of the seventh chakra, that was normal (rated in eight). The Chinese medicine's diagnosis of this patient was Blood deficiency, Kidney Yang and Yin deficiency. In the first appointment, the patient was presenting shortness of breath when placed lying down for physical examination and had a purplish color at the fingertips and in the lips. It was difficult to lay the patient down on the stretcher because she was more purplish and more breathless to do the first acupuncture session.

Besides the acupuncture session, I orientate her some foods that she needs to avoid to do not imbalance even more the energy of the patient. The dietary orientations were to avoid the ingestion of cold water, raw food, dairy products, and sweets. The second group of foods that I usually orientates to avoid is the ingestion of coffee, soda and mate tea. And the third group of foods that I orientate to avoid are fried foods, eggs, chocolate, honey, coconut, alcoholic beverages, melted cheese. The explanations of the reason to avoid these kinds of foods will be discussed in the discussion section. The third tool used by the author in the treatment of this specific patient was to replenish the chakras' energy centers with the use of highly diluted medications such as homeopathy medicines, according to the theory created by me (2020) entitled Constitutional Homeopathy of the Five Elements Based on Traditional Chinese Medicine and the use of crystal-based medications. The homeopathy medications were prescribed as single dose of each medication, according to the sequence of the Generation Cycle of the Five Elements theory. In this specific case, the author begin to treat with Silicea (to tone the Lung) and secondly, the physician prescribed Natrum muriaticum (to tone the Kidney), and thirdly the patient received Phosphorus to tone the Liver and after, the patient received Sulphur to tone the Heart and the last medications used was Calcarea carbonica, to tone the Spleen. The use of this kind of medication should be during a period of one year or more.

\section{Result}

After the first acupuncture session and the beginning of the use of medications (homeopathies and crystals), the patient improved from her pain in the upper limbs but what was more impressive in her treatment was that she improved very much from her dyspnea and her purpleness on the fingertips and lips that disappeared completely after beginning to treat the energy deficiency state. The focus of the treatment was not to recover the symptoms of congestive heart failure (shortness of breath and purplish color at the fingertips) but as the author treated this patients and all her patients at the root of the problem, that are the energy deficiencies, all the patients symptoms improved at the same time, even the focus of the treatment done were not to improve the Heart, but when treating the root, all the patient emotional and physical symptoms improved at the same time, even the doctor does not know that the patient has such symptom. After the treatment done, she is no longer turning purple at the extremities when lying down, she is more active, managing to do things, smiling more, she is understanding what is being said, in addition to the Heart, that is also improving. It is already being possible to reduce the dosages of some medications that she was using.

\section{Discussion}

In the treatment of this patient, I used the concepts of root treatment instead of leaf level treatment, and I will tell you briefly the case that became the cornerstone of all my treatment nowadays. This case was a 70 years-old-male patient that was treating pain in the legs for the last 6 months without any improvement using antiinflammatory medications. This patient started treatment using Chinese dietary counseling, acupuncture, apex ear bloodletting and after 10 acupuncture session, he returned to my clinic and said that he improved from his pain in the legs but also, improved from 
another condition that he did not tell me that was his glaucoma condition (that he did not reported to me in the beginning of his treatment). For the first time of his life, his intra-ocular pressure reduced from 40 to $17 \mathrm{mmHg}[5,6]$.

This case was published in quite all articles written by me to show how is the different levels (leaf and root level) perspectives and if the physician treats the root level, instead of leaf level alone, this treatment focusing on the energy imbalances on the root of the tree can treat all the patients diseases at the same time, even the doctor does not know that the patient has such symptoms $[5,6]$. To introduce some concepts of traditional Chinese medicine, I would like to explain the reasoning used through the tree like figure, to show the different perspectives between Western and traditional Chinese medicine. Western medicine thoughts comprehend the part that are visible by the naked eye, with the branches being the different medical specialties and the leaves representing the symptoms and diseases treated by each medical specialty. So, in this case, the congestive heart failure symptoms will be treated by the branch of the cardiology, in the leaf level. Otherwise, according to traditional Chinese medicine's point of view, all manifestation of symptoms presented by each patient is caused by energy imbalances that is occurring on the root of the tree, that are composed on two main theories such as Yin and Yang and the Five Elements theory, as you can see in the (Figure 1). The energy imbalances in these two theories is caused by the emotional factors and the wrong eating habits that could induce more energy imbalances of one element or a combination of energy imbalances in other organs, demonstrated in many articles published by me, such as in the article write by me (2020) entitled The Importance of Correcting Energy Imbalances in the Prevention and Treatment of Myocardial Infarction [7].

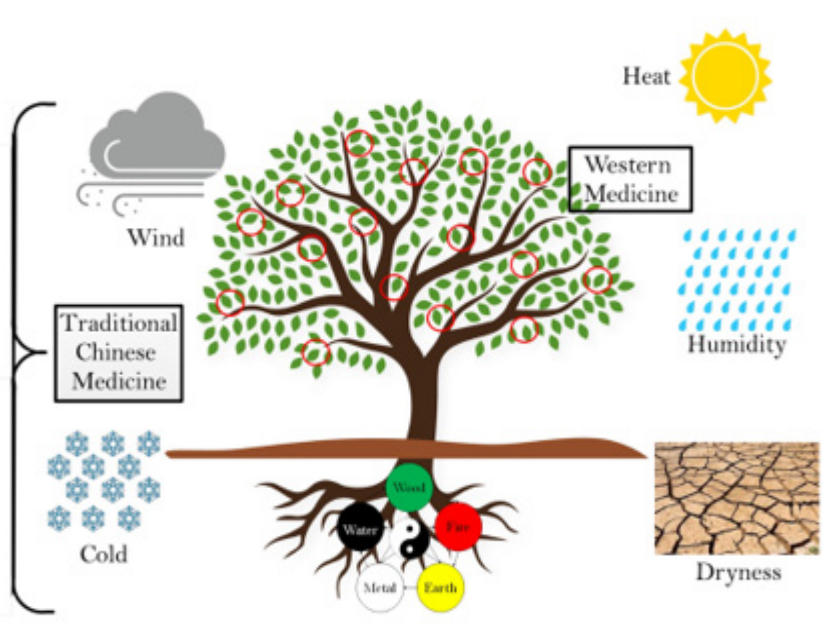

Figure 1: Tree metaphor showing the different perspectives between Western and in traditional Chinese medicine.

So, all the manifestation of symptoms in the leaf of the tree are only the symptoms. But the real cause is under the ground that we cannot see by the naked eyes, that are the imbalances of the Yin and Yang energy and the Five Elements theory, showed in the (Figure 1). So, in this case, we can understand that the Heart is not working properly due to the condition of lack of energy inside the Heart organ and all five internal massive organs, because one organ depends on the energy of the other organ and in the case reported in this article, the patient did not have energy in all five internal massive organs, needing to replenishing the energy of the entire system, to improve all the conditions of the patient, mainly the congestive heart failure symptoms, despite not being the focus of the treatment at that moment [7].

Yin and Yang are two opposite and complementary forces that exist in our world, in everything, including the human body. A possible example is day and night, men and women, good and bad. For Yin and Yang energy to flow, two other energies have to work in harmony with it that are $Q i$ and Blood [7]. Qi is basically the energy that circulates throughout your body. Qi moves the Blood, that means if Qi stays stagnant long enough, then the local body fluids that are supposed to be flowing begin to stagnate as well [7]. To achieve health, the main aim is to balance these four energies Yin, Yang, Qi and Blood, as you can see in the (Figure 2). This theory was presented in 2015 in an Acupuncture Research Conference by me in a study entitled Acupuncture Viewed Holistically Can Treat all Patients Symptoms at The Same Time, Even The Doctor Does Not Know That the Patient Has Such Symptom. This study was based on the patient with leg pain and glaucoma, presented by the author in the beginning of the discussion section [6]. The lack of energy of one of this energy or a combination of them, can lead to the formation of internal Heat inside the body, as you can see in the (Figure 3).

The second important theory that influences the way of reasoning in traditional Chinese medicine is the Five Elements theory. In this theory, they are saying that everything in the world are commended by one of these five elements that corresponds to Fire, Earth, Metal, Water and Wood. According to the article written by Chase (2018) entitled The Geometry of Emotions: Using Chakra Acupuncture and 5-Phase Theory to Describe Personality Archetypes 
for Clinical Use, he is saying that there is correlation between chakras' energy centers and the five elements in traditional Chinese medicine [8]. Each chakra energy center has correlation with each element (Wood, Fire, Earth, Metal and Water) of the Five Elements theory and each element represents one internal massive organ (Liver, Heart, Spleen, Lung, Kidney) respectively. Each internal massive organ has a hollow organ that are acoplated. For example,
Liver has Gallbladder as the hollow organ, Heart has Small intestine, Spleen has Stomach as hollow organ, Lung has Large intestine and Kidney has Bladder as hollow organ that is acoplated on it [8]. One element provide energy to the following organ in the Generation cycle and can control the functioning of the other organs, according to the Control cycle, showed in the (Figure 4) [5].

\section{Yin
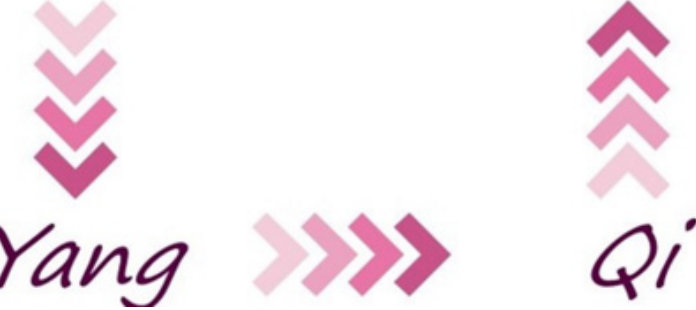

Blood

Figure 2: Yin, Yang, Qi and Blood relationship.

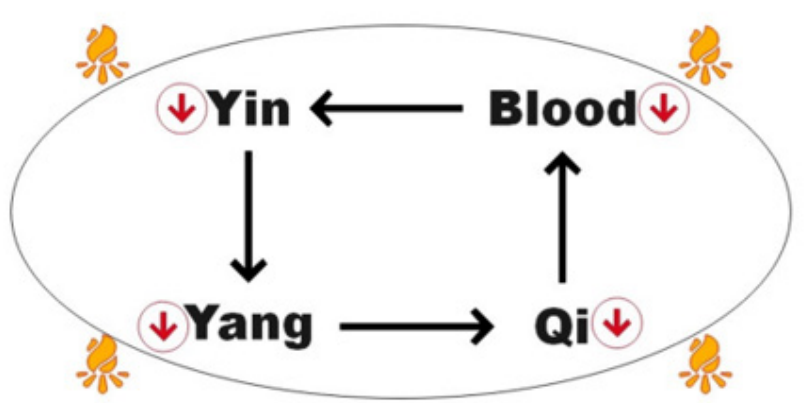

Figure 3: Internal Heat formation when there are energy deficiencies.

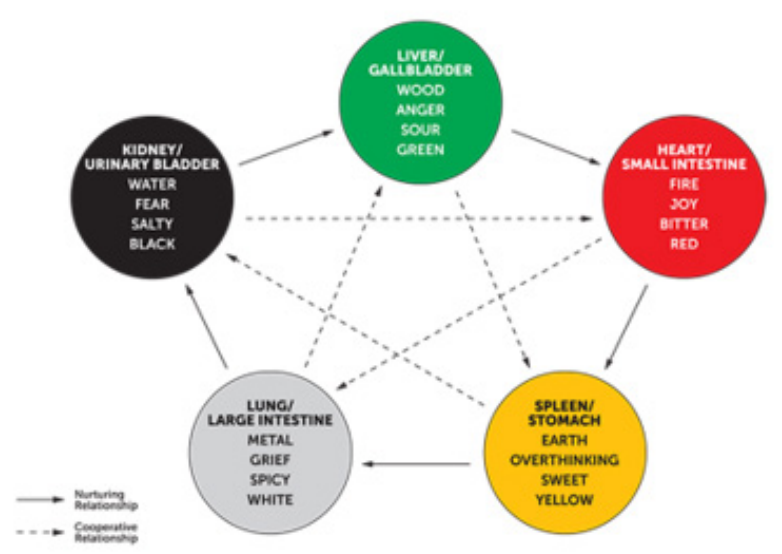

Figure 4: Five Elements theory.

The correlation between the chakras' energy centers and the internal five massive organs in traditional Chinese medicine, showed in the article written by Chase (2018), can be seeing in the (Figure 5). In this figure, I am demonstrating that one chakra has a correspondence to one internal massive organ and each massive organ is responsible for the production of one specific energy to maintain our health, as you can see in the (Figure 5). In this Figure, the chakra in red, is correspondent to the Wood element or Liver. The second chakra in orange is correspondent to the Kidney. The third chakra in yellow is correspondent to the Fire element and 
represents the Heart. The fourth chakra in green is correspondent to the Metal element and represents the Lung. The blue chakra (fifth) represents the Earth element and is correlated to the Spleen. The sixth chakra is correlated to the second chakra (Kidney) and the seventh chakra is correlated to the first chakra or Wood element or Liver $[8,9]$.

The production of Yin, Yang, Qi and Blood energy, shown in the (Figure 2), is made by one internal massive organ or one specific chakra, showed in the (Figure 5). For example, the production of Yin and Yang energy is made by the Kidney (second chakra), the production of Blood is made by the Earth element or Spleen (fifth chakra) and the distribution of $Q i$ to the entire body is made by Liver and Lung (first and fourth chakra), as you can see in the (Figure 5) [5]. According to TCM, the top six syndromes of patients with Heart failure are: Yin, Yang and Qi deficiency, Blood stasis, Water retention and turbid Phlegm. Blood stasis is found to be the main syndrome element of Heart disease, followed by Qi deficiency [10].

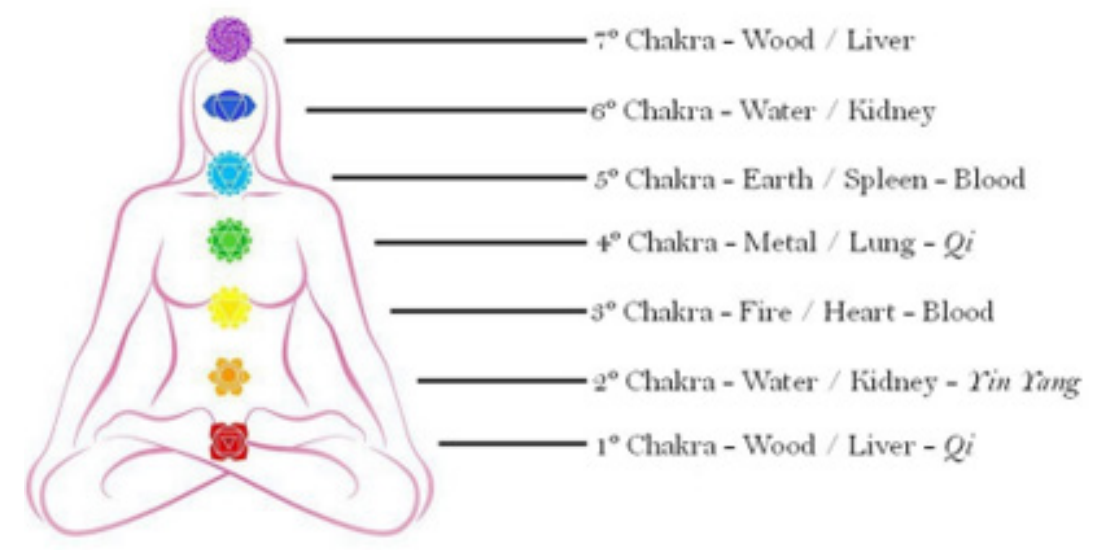

Figure 5: Chakra's energy centers and correlation between the five elements, the five internal massive organs and energy production by each organ.

Each internal massive organ has their Yin, Yang, Qi and Blood and they need to be in a balance state to keep the health of the organ [9]. In the case of the Heart Qi, this energy maintains Blood circulation, whereas Heart Yang promotes fluid metabolism. The fundamental dysfunction in Heart failure is the long period of deficiency in Heart Qi and Yang, which consequently lead to stasis of Blood circulation, as you can see in the (Figure 6) [10]. Therefore, the treatment principles are that they collectively benefit the $Q i$ and warm Yang of the Heart- specifically by activating Blood circulation to dissipate Blood stasis and disperse swelling [5]. In many articles written by me, I am showing that many times, the insufficiency of the organ function is caused by the lack of energy of the chakras' energy centers that commands the functioning of that specific organ. For example, in the article written by me (2021) entitled
Why Some Patients Do Not Get Better with the Drugs They Use to Treat Endocrine Disorders, I am saying that many times, the reduced organ function is not related to the lack of the hormone production by the organ itself but due to reduced energy in the chakra, that is responsible for sending energy to this specific organ, causing in this case, reduced or no energy to that organ or gland to work properly, leading in this case, to reduced hormones production and causing the symptoms of reduction of that specific gland function. But many time, when I begin to replenish the energy of all the chakras' energy centers, that are very weak in energy, the organ or gland begin to work again, producing the hormones that they were not producing, but due to replenishment of the energy that was lacking to them to work adequately [11].

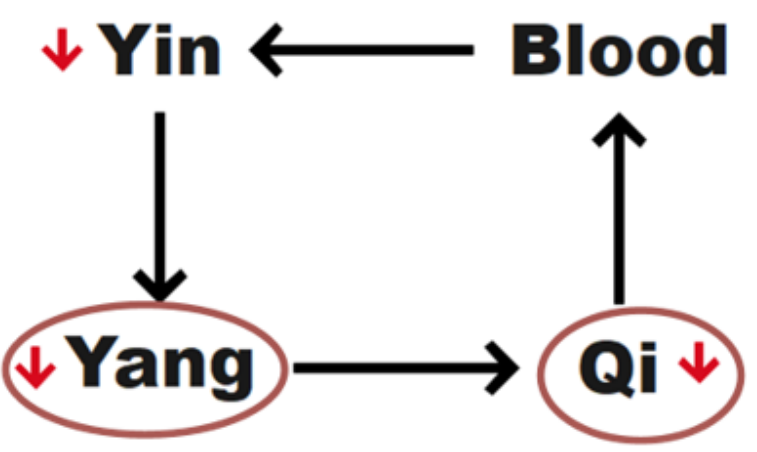

Figure 6: Relationship between Heart Qi and Yang, leading to Blood stagnation. 
In this article, I am demonstrating that patients with congestive Heart failure also have chakras' energy centers deficient in energy, that were leading to no condition to the Heart work properly. When the patient receives energy to fulfill the battery that commands the Heart function (that is the third chakra), the Heart begin to have condition to work normally again and the patient begin to recover from their initial condition, that was demonstrated in this case reported in this article. As the author showed in the beginning of the discussion section, all clinical manifestation of patients is in the leaf level of the tree but the real cause is not at the leaf, but on the root of the tree, that is usually not seeing by the naked eyes, because they are under the earth $[5,11]$. The factors that can contribute to induce energy imbalances in the root level could be emotional factors and wrong eating habits. For this reason, the first step usually made by me in all kinds to treatments is first, change the dietary aspects according to the energy imbalances presented by each patient and according to energy of each kind of food (Cold, Neutral, Warm and Hot). As said by Hippocrates (460 bce - 375 bce), make your food your medicine and your medicine your food [3].
The second step used by me was performing auricular acupuncture with apex ear bloodletting. Using this procedure, I can balance the energy of Yin, Yang, Qi and Blood, using auricular mustard seeds in the ear, as you can see in the (Figure 7). The meaning of the function of each point used is well explained in the article written by me (2018) entitled How Do You Treat Back Pain in Your Practice? Part 2 [12]. The reason why Western medicine do not study this part of the tree (root) could be explained by the implantation of Flexner report in 1910. At that time, all medical schools in United States and in Canada was reformulated and only schools that meet the criteria implanted by Flexner could still maintain open. All this reformulation leads to an improvement of the researcher in medicine but negative effects were also seeing. For example, after this implantation, they consider only what they could proof by laboratory exams or at radiological level as scientific and all kinds of medicine that works with more holistic approach, were consider as unscientific, such as the use of herbs, homeopathy, chiropractic, naturopathy, etc. [13].

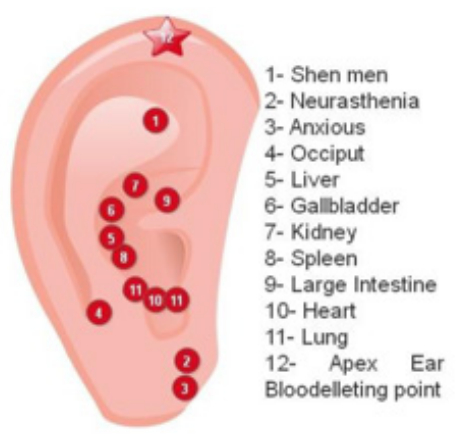

Figure 7: Auricular acupuncture points used in the treatment of patient with congestive heart failure.

But Flexner was not medical doctor and was only teacher in a former school and for this reason, he cannot know what would be important for the formation of a good medical doctor because the lack of understanding that the human being is formed by energy (and it is invisible by the naked eyes) makes medicine nowadays doing diagnosis only in the phase four and five, in a very late phase, because they cannot understand all the energy alterations that are happening in the root level, before materializing in the leaf level of the tree [13]. In this article, I am demonstrating that all the clinical manifestation of this patient, such as congestive Heart failure, Alzheimer diseases, depression and hypertension is caused by the same source, that are deficiency of energy in the five internal massive organs (Liver, Heart, Spleen, Lung and Kidney), as in the article I wrote (2021) entitled Energy Alterations and Chakras' Energy Deficiencies in Dementia Patients and in the article Energy Alterations as the Underlying Cause of Primary Hypertension. The article about depression is in process of publication entitled Why Patients with Depression Do Not Improve the Symptoms with Antidepressant Medications? where I am explaining that patients with depression diagnosis have chakras' energy centers deficient in energy that is leading to misinterpretation of the symptoms as depression, caused by the lack of neuro-transmitters $[14,15]$.

This lack of energy was diagnosed through the measurement of the chakras' energy centers, studied by Ayurvedic medicine. I am using the measurement of the chakras' energy centers to access the energy of the internal massive organs, because there are some studies from the literature such as the study written by Chase (2018) entitled The Geometry of Emotions: Using Chakra Acupuncture and 5-Phase Theory to Describe Personality Archetypes for Clinical Use are correlating chakras' energy centers with the five internal massive organs [8]. Although Heart failure is at the leaf level in the tree, the Heart is one of the five massive organs, so for it to function properly it needs energy. But the Heart depends on the energy of the Liver, that depends on the energy of the Kidney. And the Kidney depends on the energy of the Lung that depends on the energy of the Spleen, as I am showing in the Generation Cycle of the Five Elements theory, in the (Figure 4). For this reason, to treat the Heart, I need to treat the entire system that is very weak in energy and for this reason, I am also showing that the congestive Heart failure is a clinical symptom of systemic general energy deficient 
state and there is the necessity of treating the whole system and not only the Heart itself [5, 7].

The importance of measuring the chakras' energies centers is that each internal massive organ is responsible for the production of the one specific energy such as Yin, Yang, Qi and Blood, that are the four energies important to maintain our health. Yin and Yang are produced by the Kidney ( $2^{\text {nd }}$ chakra). Blood is produced by the Spleen ( $5^{\text {th }}$ chakra) and the flow of Blood inside the vessels is commanded by the Heart ( $3^{\text {rd }}$ chakra). The distribution of $Q i$ is the responsibility of the Liver (1st chakra) and Lung ( $4^{\text {th }}$ chakra), as you can see in the (Figure 5) [5]. The author used to treat this energy deficiency state of the five internal massive organs using highly diluted medications such as homeopathy, as showed in the (Table 1 ). The medications used is according to the theory created by me (2020), entitled Constitutional Homeopathy of the Five Elements based on Traditional Chinese Medicine when I begin to study homeopathy in Brazil in 2015. This kinds of medications need to be used for one year or more but the author, through her clinical experiences, is felling that the use of this kind of medication should be used to the entire life of the patient because it is a kind of energy that you need to fulfill the energy of the internal organs, because the human body is constantly exposed to this kinds of radiation globally, due to the modernization of the telecommunication, as showed in the article written by me (2021) entitled The Influence of Cell Phones and Computers on Our Immune System [16, 17].

Table 1: Homeopathy medications used to treat the energy deficiency state of the five internal massive organs in TCM.

\begin{tabular}{|l|c|c|c|}
\hline Chakras & Five Elements & Homeopathy Medications \\
\hline $1^{\circ}$ Chakra & Wood / Liver & Phosphorus \\
\hline $2^{\circ}$ Chakra & Water / Kidney & Natrum muriaticum & Sulphur \\
\hline $3^{\circ}$ Chakra & Fire / Heart & Silicea \\
\hline $4^{\circ}$ Chakra & Metal / Lung & Calcarea carbonica calcite \\
\hline $5^{\circ}$ Chakra & Earth / Spleen & Tone $2^{\circ}$ chakra \\
\hline $6^{\circ}$ Chakra & Water / Kidney & Tone $1^{\circ}$ chakra \\
\hline $7^{\circ}$ Chakra & Wood / Liver & Blue Quartz \\
\hline
\end{tabular}

In China, when patients have energy deficiency in the five internal massive organs, they usually prescribe Chinese herbs, but this kind of medication is more difficult to find in Brazil. What I found after this study is that homeopathy is a type of medication that works very well in the treatment of many symptoms and diseases. According to Hahnemann (1755 - 1843), the German doctor who created homeopathy, this kinds of medications works according to the theory of simmilimum. For example, when you give one kind of substance for one patient and he becomes sick after the ingestion of this substance, the medication that will treat this condition is the same substance given in a very diluted way [18].
This is the type of medication that I am treating patients with SARSCoV-2 infection, because as I showed in another article, not only patient with congestive heart failure that has energy deficiency in the five internal massive organs but all kinds of patients that has chronic diseases such as depression, anxiety, panic syndrome, diabetes, hypertension, myocardial infarction, stroke, cancer, etc, what all have in common are chakras' energy centers deficient in energy, caused by the exposition to the electromagnetic radiation, showed in the article I wrote (2021) entitled Energy Alterations and Chakras' Energy Deficiencies and Propensity to SARS-CoV-2 Infection [19].

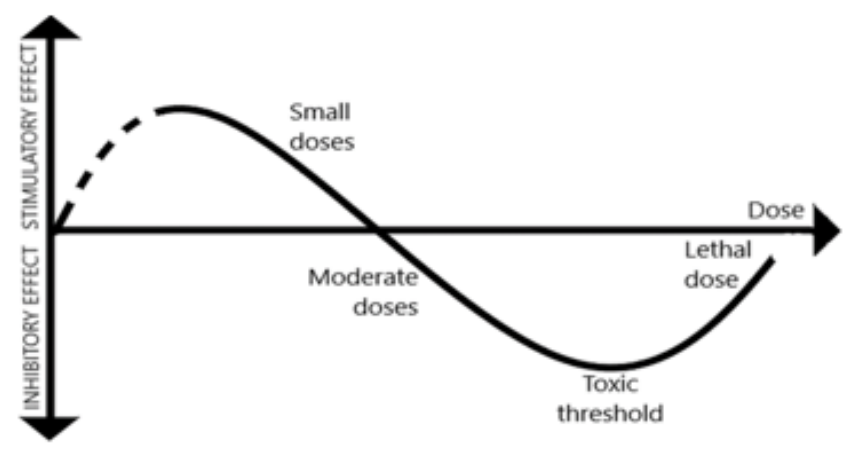

Figure 8: Arndt-Schultz Law.

The use of highly concentrated medications such be used with precautions nowadays, because the pattern of internal energy of our population changed, as I am demonstrating in the article Is the Population in the World the Same as in the Past? In another article in process of publication by me (2021) entitled Why Is Homeopathy the Medication of Choice in the Treatment of All Types of Illnesses Nowadays? Through both articles, I am saying that the use of highly diluted medications are the drugs to choice nowadays because 
when using highly concentrated medications, according to ArndtSchultz Law, created by two German researchers, in 1888, they are saying that highly concentrated medications can harm even more the vital energy of the patient and can have toxic effect or lead to dead, as you can see in the (Figure 8). That is why the drugs of choice nowadays, are highly diluted medications, to improve the vital energy of the patients, that is already low, demonstrated in the article written by me (2021) entitled Energy Alterations and Chakras' Energy Deficiencies and Propensity to SARS-CoV-2 Infection and in another article also written by me (2021) entitled Are We Vaccinating Immunocompetent or Immunocompromised People for COVID 19? [20-22]

It is important to say that homeopathy was recognized by the Federal Council of Medicine in Brazil in 1980 and by the Council of Medical Specialties of the Brazilian Medical Association in 1990.
And the acupuncture was recognized by the Federal Council of Medicine in Brazil in 1995. As they were not considered scientific drugs since Flexner implantation, but with the modernization of telecommunication, drugs that was considered nonscientific are the drugs of choice nowadays, and drugs considered scientific (highly concentrated medications) are causing harm to the patients, due to the energy reduction state [23]. To finalize this article, I would like to show this metaphor between Yin and Yang Theory to show that the two kinds of medicine (Western and traditional Chinese medicine) are different but are complementary, as I am showing in the (Figure 9). The importance to understand that all the things is made by energy, including the human being, need to be teached in all medical schools around the world, to the doctors begin to understand the formation of disease since the energy alteration and not only after materializing, as it is done by Western medicine nowadays [24].

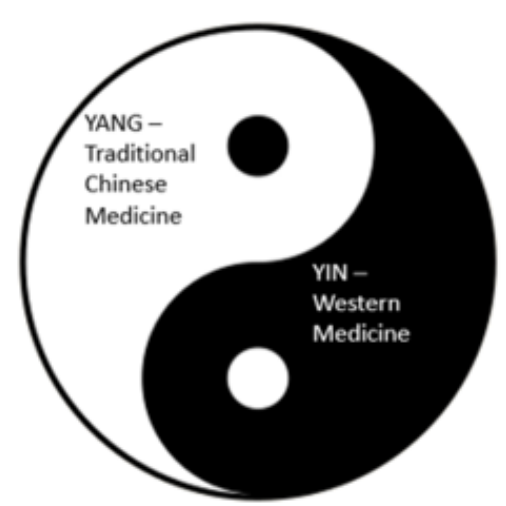

Figure 9: Integration of Western and Traditional Chinese Medicine.

\section{Conclusion}

The conclusion of this study is that patients with congestive heart failure have energy deficiencies in the chakras' energies centers and the treatment correcting the energies disturbances using Chinese dietary counselling, auricular acupuncture and apex ear bloodletting and replenishing the chakras' energy centers using homeopathies medications according to the theory Constitutional Homeopathy of the Five Elements Based on Traditional Chinese Medicine and crystal-based medications are very important tools in the treatment of patients with this pathology because we will be treating the cause of the disease formation and not only treating the symptoms.

\section{Acknowledgement}

None.

\section{Conflict of Interest}

No conflict of interest.

\section{References}

1. Inamdar A, Inamdar A (2016) Heart Failure: Diagnosis, Management and Utilization. J Clin Med 5(7): 62.
2. Healthwise Staff (2020) Heart Failure Symptoms.

3. Craik E (2014) The "Hippocratic" Corpus: Content and Context. Routledge p. 344.

4. Xiao-Qian Li, Jian-Cheng He, Pin-Xian Huang, Xue-Bin Cao (2016) Chinese medicine syndromes in congestive heart failure: A literature study and retrospective analysis of clinical cases. Chin J Integr Med 22(10): 738-744.

5. Huang WL (2019) Why Are Diabetic Patients Still Having Hyperglycemia despite Diet Regulation, Antiglycemic Medication, and Insulin. Int J Diabetes Metab Disord 4(2): 1-14.

6. Huang Wei Ling (2019) The Importance of Correcting Energy Imbalances and Chakras Energy Deficiencies in the Treatment of Patients with Glaucoma. Clin Res Ophthalmol 2(2): 1-9.

7. Huang Wei Ling (2020) The Importance of Correcting Energy Imbalances in the Prevention and Treatment of Myocardial Infarction. Acta Scientific Medical Sciences 4(6): 20-27.

8. Chase CR (2018) The Geometry of Emotions: Using Chakra Acupuncture and 5-Phase Theory to Describe Personality Archetypes for Clinical Use. Med Acupunct 30(4): 167-178.

9. Ni M (1995) Yellow Emperor's Classic of Medicine: The Essential Text of Chinese Health and Healing. First. Shambhala, Boston and London.

10. Yong Wang, Qiyan Wang, Chun Li, Linghui Lu, Qian Zhang, et al. (2017) A Review of Chinese Herbal Medicine for the Treatment of Chronic Heart Failure. Curr Pharm Des 23(34): 5115-5124. 
11. Huang WL (2021) Why Some Patients Do Not Get Better with the Drugs They Use to Treat Endocrine Disorders. SunText Rev Endocrine Care 1(1): 103.

12. Huang Wei Ling (2018) How Do You Treat Back Pain in Your Practice? Part 2. Medical Acupunture 30(1): 46-53.

13. Huang Wei Ling (2021) What Flexner Report did to Our Medicine After 100 Years of Implantation. Acta Scientific Gastrointestinal Disorders 4(8): 1-04.

14. WL Huang (2021) Energy Alterations and Chakras' Energy Deficiencies in Dementia Patients. J Neurol Sci Res 2(1): 1-30.

15. Huang Wei Ling (2019) Energy Alterations as the Underlying Cause of Primary Hypertension. ARC J Nephrol 4(2): 33-44.

16. Huang Wei Ling (2020) Constitutional Homeopathy of Five Elements Based on Traditional Chinese Medicine. Acta Scientific Medical Sciences 4(7): 57-69.

17. Huang WL (2021) The Influence of Cell Phones and Computers on Our Immune System. Ann Immunol Immunother 3(2): 000141.
18. Why Homepathy is the Medication of Choice in the Traetment of Sars Cov 2 Infection?

19. Huang Wei Ling (2021) Energy Alterations and Chakras' Energy Deficiencies and Propensity to SARS-CoV-2 Infection. Acta Scientific Microbiology 4(4): 167-196.

20. Huang Wei Ling (2021) Is the Population in the World the Same as in the Past. Acta Scientific Clinical Case Reports v. 2(6).

21. (2020) Arndt Schultz Law and its applications in Homeopathy.

22. Huang WL (2021) Are We Vaccinating Immunocompetent or Immunocompromised People for COVID 19. J Vaccines Res Vaccin 7: 018.

23. Huang Wei Ling (2021) Are The Medications That We Are Prescribing To Our Patients Harming Them. 6(1): 11-13.

24. Huang Wei Ling (2018) Why Do Patients Still Catch Hospital Infections Despite the Practice of Infection Prevention and Control Programs. Acta Scientific Microbiol 1(4): 34-43. 